\title{
PROPER HEREDITARY SHAPE EQUIVALENCES PRESERVE SMALL WEAK INFINITE DIMENSIONALITY
}

\author{
RICHARD P. MILLSPAUGH
}

(Communicated by James E. West)

\begin{abstract}
A space is said to be small weakly infinite dimensional if it has a basis $\mathscr{B}$ such that the collection of finite unions of elements of $\mathscr{B}$ is inessential. A characterization of small weak infinite dimensionality is given for locally compact spaces. This characterization is then used to prove that if $f: X \rightarrow Y$ is a proper hereditary shape equivalence from a metric space $X$ which is small weakly infinite dimensional onto a locally compact metric space $Y$, then $Y$ is small weakly infinite dimensional.
\end{abstract}

\section{INTRODUCTION AND DEFINITIONS}

Small weak infinite dimensionality is a property first introduced by $\mathrm{P}$. Borst in [B1] and further studied in [B2]. The purpose of this paper is to study the preservation of small weak infinite dimensionality by proper hereditary shape equivalences. The corresponding problem for Property $\mathrm{C}$ is investigated by F. D. Ancel in [A1] and for weak infinite dimensionality in the sense of Alexandroff by $D$. Rohm in [R]. In order to prove our main result, we first obtain a useful characterization of small weak infinite dimensionality for locally compact spaces.

All spaces are assumed to be metric. We begin by stating several definitions:

1.1. Definition. A collection $\mathscr{C}$ of subsets of a space $X$ is said to be inessential if for every sequence $\left\{\left(A_{i}, B_{i}\right)\right\}_{i=1}^{\infty}$ of pairs of elements of $\mathscr{C}$ with disjoint closures there exist open subsets $U_{i}, i=1,2,3, \ldots$, of $X$ such that

$$
A_{i} \subset U_{i} \subset \operatorname{cl}\left(U_{i}\right) \subset X \backslash B_{i} \text { for each } i
$$

and so that

$$
\bigcap_{i=1}^{n} \operatorname{bd}\left(U_{i}\right)=\varnothing \text { for some positive integer } n \text {. }
$$

Received by the editors March 5, 1988 and, in revised form, September 5, 1989; presented to the 838th meeting of the American Mathematical Society on November 14, 1987.

1980 Mathematics Subject Classification (1985 Revision). Primary 54F45; Secondary 54C10.

Key words and phrases. Small weakly infinite dimensional, hereditary shape equivalence, approximately invertible map, inessential.

This work forms part of the author's doctoral dissertation, the research for which was directed by L. R. Rubin. 
1.2. Definition. A space $X$ is said to be small weakly infinite dimensional, abbreviated small-wid, if there exists a basis $\mathscr{B}$ for the topology on $X$ with the property that $\mathscr{B}^{\text {fin }}$ is inessential, where $\mathscr{B}^{\text {fin }}$ denotes the collection of finite unions of elements of $\mathscr{B}$.

As in [A1], to obtain our results we use the fact that proper hereditary shape equivalences are also approximately invertible proper surjections.

1.3. Definition. A map $f: X \rightarrow Y$ is said to be approximately invertible if there is a closed embedding $i: X \rightarrow Z$ of $X$ into a space $Z$ such that if $\mathscr{W}$ is any cover of $i(X)$ by open subsets of $Z$ which is rufined by the collection $\left\{i\left(f^{-1}(y)\right) \mid y \in Y\right\}$, then there is a map $g: Y \rightarrow Z$, called a $\mathscr{W}$-approximate inverse for $f$, such that $d(g \circ f, i)<\mathscr{W}$.

Note. When $f: X \rightarrow Y$ is approximately invertible, it will greatly simplify notation to assume that $X$ is a closed subset of $Z$ and that the inclusion map $i: X \hookrightarrow Z$ is the embedding, realizing the approximate invertibility of $f$. In this case, the cover $\mathscr{W}$ must be refined by the collection of point inverses of $f$.

\section{A CHARACTERIZATION OF SMALL WEAK INFINITE DIMENSIONALITY}

In the present section we will obtain a characterization for small weak infinite dimensionality which holds for locally compact spaces. This characterization will prove useful in obtaining the main theorem in the sequel.

2.1. Lemma. For a locally compact space $X$, the following are equivalent:

(1) $X$ is small-wid.

(2) The collection of compact subsets of $X$ is inessential.

Proof. (1) $\Rightarrow(2)$. Since each pair of disjoint compact subsets of $X$ can be separated by a pair of elements of $\mathscr{B}^{\text {fin }}$ with disjoint closures for any basis $\mathscr{B}$, this implication follows immediately from Definitions 1.1 and 1.2. Note that local compactness is not used for this part of the proof.

$(2) \Rightarrow(1)$. Since $X$ is locally compact, there is a basis $\mathscr{B}$ for $X$ such that the elements of $\mathscr{B}$ have compact closures. Note that since each element of $\mathscr{B}^{\text {fin }}$ has compact closure, the collection of closures of elements of $\mathscr{B}^{\text {fin }}$, hence also $\mathscr{B}^{\text {fin }}$, is inessential by hypothesis.

In addition to the above lemma characterizing small weak infinite dimensionality, we will require the following technical lemma:

2.2. Lemma. Let $f: X \rightarrow Y$ be an approximately invertible surjection. We assume as noted after Definition 1.3 that $X$ is a closed subset of $Z$, etc. Let $\left\{\left(A_{i}, B_{i}\right)\right\}_{i=1}^{n}$ be a finite sequence of pairs of subsets of $Y$ with disjoint closures such that there exist open subsets $V_{i}$ of $Z, i=1,2, \ldots, n$, with

$$
f^{-1}\left(\operatorname{cl}\left(A_{i}\right)\right) \subset V_{i} \subset \operatorname{cl}\left(V_{i}\right) \subset Z \backslash f^{-1}\left(\operatorname{cl}\left(B_{i}\right)\right) \text { for each } i
$$


and

$$
\bigcap_{i=1}^{n} \mathrm{bd}\left(V_{i}\right)=\varnothing .
$$

Then there exists a cover $\mathscr{W}$ of $X$ by open subsets of $Z$ and a $\mathscr{W}$-approximate inverse $g: Y \rightarrow Z$ for $f$ such that

$$
\operatorname{cl}\left(A_{i}\right) \subset g^{-1}\left(V_{i}\right) \subset \operatorname{cl}\left(g^{-1}\left(V_{i}\right)\right) \subset Y \backslash \operatorname{cl}\left(B_{i}\right) \text { for each } i
$$

and

$$
\bigcap_{i=1}^{n} \operatorname{bd}\left(g^{-1}\left(V_{i}\right)\right)=\varnothing .
$$

Proof. First note that if $\mathscr{U}$ and $\mathscr{V}$ are covers of $X$ by open subsets of $Z$ each of which is refined by the collection of point inverses of $f$, and if $\mathscr{U}$ refines $\mathscr{V}$, then any $\mathscr{U}$-approximate inverse for $f$ is also a $\mathscr{V}$-approximate inverse for $f$.

We will construct a finite sequence of covers $\mathscr{W}_{i}$ of $X$ by open subsets of $Z, i=1,2, \ldots, n$, which satisfy the following:

(a) $\mathscr{W}_{i}$ is refined by the collection of point inverses of $f$ for each $i$,

(b) $\mathscr{W}_{i+1}$ refines $\mathscr{W}_{i}$ for each $i=1,2, \ldots, n-1$, and

(c) for every $\mathscr{W}_{i}$-approximate inverse $g_{i}: Y \rightarrow Z$ for $f$ we have

$$
\operatorname{cl}\left(A_{i}\right) \subset g_{i}^{-1}\left(V_{i}\right) \subset \operatorname{cl}\left(g_{i}^{-1}\left(V_{i}\right)\right) \subset Y \backslash \operatorname{cl}\left(B_{i}\right) .
$$

Property (a) will assure that there exists a $\mathscr{W}_{i}$-approximate inverse $g_{i}: Y \rightarrow Z$ for $f$ for each $i$. Property (b) will guarantee that any $\mathscr{W}_{n}$-approximate inverse is also a $\mathscr{W}_{i}$-approximate inverse for each $i$. Finally, (c) will assure us of having

$$
\operatorname{cl}\left(A_{i}\right) \subset g_{n}^{-1}\left(V_{i}\right) \subset \operatorname{cl}\left(g_{n}^{-1}\left(V_{i}\right)\right) \subset Y \backslash \operatorname{cl}\left(B_{i}\right) \text { for each } i \text {. }
$$

Before constructing the open covers $\mathscr{W}_{i}$ as stated above, we construct an intermediate sequence of covers $\mathscr{U}_{i}$ of $X$ by open subsets of $Z$ which satisfy (a) and (c) only. We proceed as follows. For each $i=1,2, \ldots, n$, we define

$$
\begin{aligned}
U_{i} & =Z \backslash \operatorname{cl}\left(V_{i}\right), \\
W_{i} & =Z \backslash\left[f^{-1}\left(\operatorname{cl}\left(A_{i}\right)\right) \cup f^{-1}\left(\operatorname{cl}\left(B_{i}\right)\right)\right],
\end{aligned}
$$

and

$$
\mathscr{U}_{i}=\left\{U_{i}, V_{i}, W_{i}\right\} .
$$

Clearly, $\mathscr{U}_{i}$ satisfies (a). We will show that $\mathscr{U}_{i}$ also satisfies (c).

Let $h: Y \rightarrow Z$ be any $\mathscr{U}_{i}$-approximate inverse for $f, a \in \operatorname{cl}\left(A_{i}\right)$, and $x \in$ $f^{-1}(a)$. By definition we have $x \notin U_{i} \cup W_{i}$, so it must be the case that $x \in V_{i}$. Since $h$ is a $\mathscr{U}_{i}$-approximate inverse for $f$, it follows that $\{x, h(f(x))\} \subset V_{i}$. Therefore, $h(f(x))=h(a) \in V_{i}$ and we have that

$$
\operatorname{cl}\left(A_{i}\right) \subset h^{-1}\left(V_{i}\right) \text {. }
$$


In a similar fashion, one shows that

$$
\operatorname{cl}\left(B_{i}\right) \subset h^{-1}\left(U_{i}\right)=h^{-1}\left(Z \backslash \operatorname{cl}\left(V_{i}\right)\right) .
$$

Since we have

$$
h^{-1}\left(\operatorname{cl}\left(V_{i}\right)\right) \cap h^{-1}\left(Z \backslash \operatorname{cl}\left(V_{i}\right)\right)=\varnothing
$$

and

$$
\operatorname{cl}\left(h^{-1}\left(V_{i}\right)\right) \subset h^{-1}\left(\operatorname{cl}\left(V_{i}\right)\right)
$$

it follows that

$$
\operatorname{cl}\left(h^{-1}\left(V_{i}\right)\right) \subset Y \backslash \operatorname{cl}\left(B_{i}\right)
$$

Hence,

$$
\operatorname{cl}\left(A_{i}\right) \subset h^{-1}\left(V_{i}\right) \subset \operatorname{cl}\left(h^{-1}\left(V_{i}\right)\right) \subset Y \backslash \operatorname{cl}\left(B_{i}\right),
$$

and $\mathscr{U}_{i}$ satisfies $(\mathrm{c})$.

We complete the proof of the lemma by constructing the desired covers $\mathscr{W}_{i}$ inductively as follows. Let $\mathscr{W}_{1}=\mathscr{U}_{1}$ and, for each $i=2,3, \ldots, n$, let

$$
\mathscr{W}_{i}=\left\{W \cap U \mid W \in \mathscr{W}_{i-1} \text { and } U \in \mathscr{U}_{i}\right\} .
$$

Then $\mathscr{W}_{i}$ satisfies (a) for each $i$ because both $\mathscr{U}_{i}$ and $\mathscr{W}_{i-1}$ do. Further, since $\mathscr{W}_{i}$ refines both $\mathscr{U}_{i}$ and $\mathscr{W}_{i-1}, \mathscr{W}_{i}$ must satisfy both (b) and (c).

Since

$$
\bigcap_{i=1}^{n} \mathrm{bd}\left(V_{i}\right)=\varnothing
$$

and $g: Y \rightarrow Z$ is continuous, it follows that

$$
\bigcap_{i=1}^{n} \operatorname{bd}\left(g^{-1}\left(V_{i}\right)\right)=\varnothing \text {. }
$$

\section{MAIN THEOREM}

We are now prepared to investigate the preservation of small weak infinite dimensionality by proper hereditary shape equivalences when the range is locally compact. We first prove the following:

3.1. Lemma. Let $f: X \rightarrow Y$ be an approximately invertible proper surjection from a space $X$ which is small-wid onto a locally compact space $Y$. Then $Y$ is small-wid.

Proof. By Lemma 2.1, it suffices to prove that the collection of compact subsets of $Y$ is inessential. To this end, let $\left\{\left(A_{i}, B_{i}\right)\right\}_{i=1}^{\infty}$ be a sequence of disjoint pairs of compact subsets of $Y$. Then $\left\{\left(f^{-1}\left(A_{i}\right), f^{-1}\left(B_{i}\right)\right)\right\}_{i=1}^{\infty}$ is a sequence of disjoint pairs of compact subsets of $X$. Since $X$ is small-wid, the collection of compact subsets of $X$ is inessential by Lemma 2.1 (recall that this did not require local compactness), so there must exist open subsets $\widetilde{U}_{i}$ of $X, i=$ $1,2,3, \ldots$, such that

$$
f^{-1}\left(A_{i}\right) \subset \widetilde{U}_{i} \subset \operatorname{cl}\left(\widetilde{U}_{i}\right) \subset Z \backslash f^{-1}\left(B_{i}\right) \text { for each } i
$$


and

$$
\bigcap_{i=1}^{n-1} \operatorname{bd}_{X}\left(\widetilde{U}_{i}\right)=\varnothing \text { for some positive integer } n-1,
$$

where $\operatorname{bd}_{X}\left(\widetilde{U}_{i}\right)$ denotes the boundary of $\tilde{U}_{i}$ in $X$. Now use the extension defined in $[\mathrm{H}$, page 125$]$ to find open subsets $U_{i}$ of $Z, i=1,2,3, \ldots$, such that

$$
f^{-1}\left(A_{i}\right) \subset U_{i} \subset \operatorname{cl}\left(U_{i}\right) \subset Z \backslash f^{-1}\left(B_{i}\right) \text { for each } i
$$

and

$$
\bigcap_{i=1}^{n-1} \operatorname{bd}\left(\widetilde{U}_{i}\right)=\varnothing \text { for some positive integer } n-1 \text {. }
$$

Let $W$ be an open neighborhood of $X$ in $Z$ whose closure does not intersect $\bigcap_{i=1}^{n-1} \operatorname{bd}\left(U_{i}\right)$. Now replace $U_{n}$ with $W \cap U_{n}$ to get open subsets $U_{i}, i=$ $1,2, \ldots, n$ of $Z$ such that

$$
f^{-1}\left(A_{i}\right) \subset U_{i} \subset \operatorname{cl}\left(U_{i}\right) \subset Z \backslash f^{-1}\left(B_{i}\right) \text { for each } i
$$

and

$$
\bigcap_{i=1}^{n} \operatorname{bd}\left(U_{i}\right)=\varnothing
$$

We now apply Lemma 2.2 to find an approximate inverse $g: Y \rightarrow Z$ for $f$ such that

$$
A_{i} \subset g^{-1}\left(U_{i}\right) \subset \operatorname{cl}\left(g^{-1}\left(U_{i}\right)\right) \subset Y \backslash B_{i} \text { for each } i
$$

and

$$
\bigcap_{i=1}^{n} \operatorname{bd}\left(g^{-1}\left(U_{i}\right)\right)=\varnothing
$$

Setting $V_{i}=g^{-1}\left(U_{i}\right)$ for $i=1,2, \ldots, n$ and letting $V_{i}$ be any open neighborhood of $A_{i}$ whose closure does not intersect $B_{i}$ for $i=n+1, n+2, n+3, \ldots$ yields a sequence of open subsets $V_{i}$ of $Y$ such that

$$
A_{i} \subset V_{i} \subset \operatorname{cl}\left(V_{i}\right) \subset Y \backslash B_{i} \text { for each } i
$$

and such that

$$
\bigcap_{i=1}^{n} \operatorname{bd}\left(V_{i}\right)=\varnothing \text {. }
$$

Therefore, the collection of compact subsets of $Y$ is inessential and $Y$ is smallwid.

Since proper hereditary shape equivalences are also approximately invertible proper surjections [A2, Theorem 4.5] we obtain the following theorem as an 
immediate corollary to Lemma 3.1:

3.2. Theorem. Let $f: X \rightarrow Y$ be a proper hereditary shape equivalence from a space $X$ which is small-wid onto a locally compact space $Y$. Then $Y$ is small-wid.

Since proper closed maps preserve local compactness, we obtain the following as an immediate corollary to Theorem 3.2 and the fact that proper maps between metric spaces are closed:

3.3. Corollary. Let $f: X \rightarrow Y$ be a proper hereditary shape equivalence onto a space $Y$ from a locally compact space $X$ which is small-wid. Then $Y$ is small-wid.

The following two propositions are due to P. Borst [B1] and are presented here without proof.

3.4. Proposition. $A$ (topologically) complete separable metric space $X$ is smallwid if and only if every compact subspace of $X$ is small-wid.

3.5. Proposition. If a space $X$ is small-wid, then every compact subspace of $X$ is small-wid.

Applying these propositions, we obtain the following further corollary to Theorem 3.2:

3.6. Corollary. Let $f: X \rightarrow Y$ be a proper hereditary shape equivalence from a space $X$ which is small-wid onto a (topologically) complete separable metric space $Y$. Then $Y$ is small-wid.

Proof. By Proposition 3.4, it suffices to show that an arbitrary compact subspace of $Y$ is small-wid. Let $M$ be such a compact subspace of $Y$.

Claim. The restriction $f \mid f^{-1}(M): f^{-1}(M) \rightarrow M$ is an approximately invertible surjection.

Proof of claim. Clearly $f \mid f^{-1}(M): f^{-1}(M) \rightarrow M$ is a proper surjection, so we need only prove that it is approximately invertible. We make the standard assumptions that $X$ is a closed subset of $Z$, etc. Note that $f^{-1}(M)$ is a closed subset of $Z$. Let $\mathscr{W}$ be a cover of $f^{-1}(M)$ by open subsets of $Z$ such that $\mathscr{W}$ is refined by the collection of point inverses of $f$. Then we can define a cover $\mathscr{V}$ of $X$ by $\mathscr{V}=\mathscr{W} \cup\left\{Z \backslash f^{-1}(M)\right\}$. The cover $\mathscr{V}$ is refined by the set of point inverses of $f$, so there must exist a $\mathscr{V}$-approximate inverse $g: Y \rightarrow Z$ for $f$. The restriction $g \mid M: M \rightarrow Z$ is the desired $\mathscr{W}$-approximate inverse for $f \mid f^{-1}(M)$, and the claim is proved.

Proposition 3.5 implies that $f^{-1}(M)$ is small-wid, so we may apply Lemma 3.1 to complete the proof of the corollary. 


\section{REFERENCES}

[AG] D. F. Addis and J. H. Gresham, A class of infinite dimensional spaces, Part I: Dimension theory and Alexandroff's problem, Fund. Math. 101 (1978), 195-205.

[A1] F. D. Ancel, Proper hereditary shape equivalences preserve property $C$, Topology Appl. 19 (1985), 71-74.

[A2] - The role of countable dimensionality in the theory of cell-like relations, Trans. Amer. Math. Soc. 287 (1985), 1-40.

[B1] P. Borst, Spaces having a weakly-infinite-dimensional compactification, Topology Appl. 21 (1985), 261-268.

[B2] - Some remarks concerning $C$-spaces, preprint.

[H] S. T. Hu, Theory of retracts, Wayne State University Press, Detroit, 1965.

[HW] W. Hurewicz and H. Wallman, Dimension theory, Princeton University Press, Princeton, NJ, 1941.

[R] D. Rohm, Hereditary shape equivalences preserve weak infinite dimensionality, preprint.

Department of Mathematics, University of Oklahoma, Norman, Oklahoma 73019 